\title{
Rapid Growing Intramuscular Myxoma Mimicking a Peripheral Nerve Sheath Tumor
}

\author{
Homajoun Maslehaty, b, Harald Barth ${ }^{\mathrm{a}}$, Mehran Mahvash ${ }^{\mathrm{a}}$, Heinz-Hermann Hugo ${ }^{\mathrm{a}}$, \\ Hubertus Maximilian Mehdorna
}

\begin{abstract}
We present a rare case of rapid growing intramuscular myxoma mimicking a peripheral nerve sheath tumor (PNST). During diagnostic investigations for abdominal pain in a 65 -year-old female patient a $5.5 \mathrm{~cm}$ diameter tumor in the left buttock was found incidentally. The tumor was septated and hyperintense in the T2weighted images in the left gluteal region, adjacent to the sciatic nerve, presumed to be a PNST. The follow up images four months later showed marked gain of the tumor size. The tumor has been resected completely and histopathological examination revealed the diagnosis of an intramuscular myxoma. As an uncommon benign tumor, intramuscular myxoma presented very suspiciously for a PNST because of direct adjacency to the sciatic nerve with an increased growth tendency. Despite the benign entity of intramuscular myxoma surgical treatment was indicated because of the rapid growth and unclear circumstances with the possibility of malignant behavior.
\end{abstract}

Keywords: Myxoma; Intramuscular myxoma; Peripheral nerve sheath tumor; PNST

\section{Introduction}

Intramuscular myxoma is a benign tumor that presents as a slow growing deeply located mass narrowed by the skeletal muscle. The tumor usually occurs between 40 and 70 years

Manuscript accepted for publication June 17, 2011

${ }^{a}$ Department of Neurosurgery, University Hospitals Schleswig-Holstein, Campus Kiel, Germany

${ }^{\mathrm{b}}$ Corresponding author: Homajoun Maslehaty, Klinik fur Neurochirurgie,

Universitatsklinikum Schleswig-Holstein, Campus Kiel,

Arnold-Heller-Straße 3, 24105 Kiel, Germany.

Email: h.maslehaty@gmx.de

doi:10.4021/jnr22w of age and is slightly more common in females. The tumor is frequently located in the tight, upper extremity and infrequently in the buttock [1]. We present a case of a rapid growing intramuscular myxoma in the buttock with direct adjacency to the sciatic nerve, which appeared very suspicious for a peripheral nerve sheath tumor (PNST).

\section{Case Report}

A 65-year-old female patient suffered from abdominal pain since few weeks. Ultrasound, CT scanning and MRI of the lower abdomen showed bilateral ovarian cysts, witch have been resected operatively. As an incidental finding a septated tumor with a diameter of $5.5 \mathrm{~cm}$ appeared hyperintense in the T2-weighted images in the left buttock, adjacent to the sciatic nerve, presumed to be a PNST, e.g., neurinoma or neurofibroma. The follow up images four months later showed a marked gain of the tumor size to $7 \mathrm{~cm}$ (Fig. 1). The patient was admitted to our department for further therapy. The patient presented with a painless palpable swelling in the left gluteal muscle, but without evidence for sciatic nerve related neurological deficits. Due to rapid tumor growth neurosurgical resection of the tumor was indicated to obtain histopathological examination and to rule out malignancy. Intraoperatively the surface of the tumor appeared solid with an internal gelatinous and viscous fluid cyst, which was aspirated to reduce tumor size. The sciatic nerve was next to the tumor, but showed no direct connection to it. The tumor was attached to a part of the tendon of the piriformis muscle at the upper margin, assumed to be the origin. The tumor was resected completely and histopathological examination brought the diagnosis of an intramuscular myxoma (Fig. 2). The patient was discharged few days later without any complaints.

\section{Discussion}

Intramuscular myxoma usually occurs in the large skeletal muscle groups and presents as a painless slow growing swelling. Histopathological features typically contain hy- 

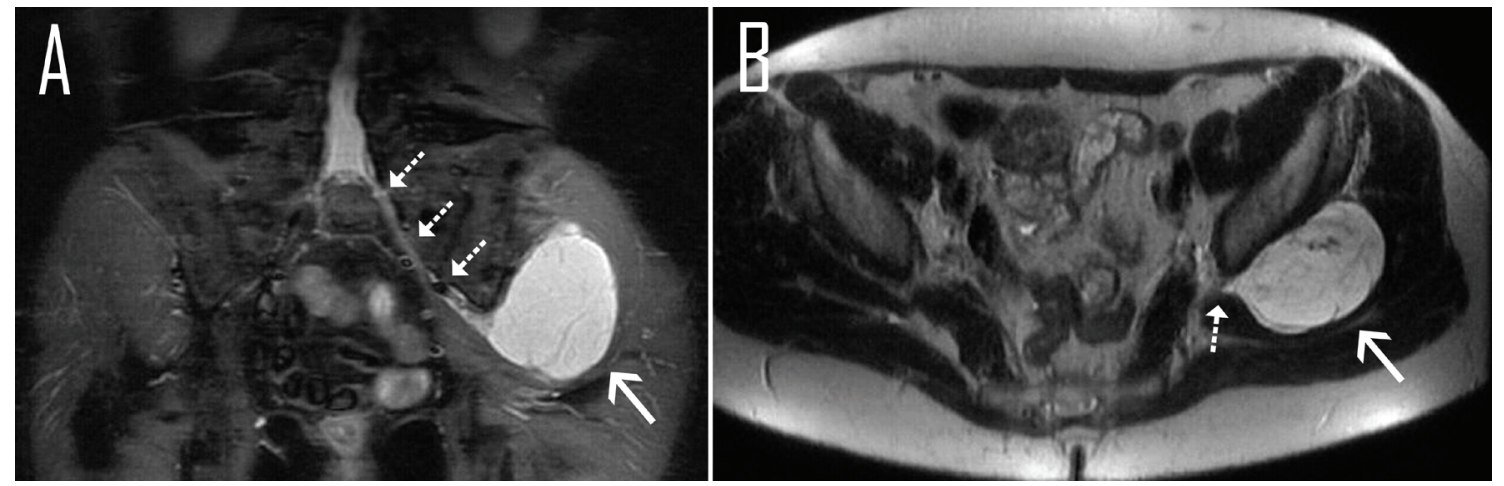

Figure 1. T2-weighted MRI of the lower abdomen, (A) coronal and (B) axial, demonstrates septated and hyperintense tumor (arrow) adjacent to the sciatic nerve (dotted arrows).

pocellularity, hypovascularity, and wing shaped fibroblasts disposed in a myxoid matrix. However, areas with increased cellularity, hypervascularization and less extracellular myxoid matrix are reported as well, which occasionally can lead to misdiagnosis of sarcoma [2]. Recurrence after surgical removal is described very infrequently, in these cases commonly with hypercellular and hypervascular areas of the tumor $[1,3,4]$.

MRI usually shows a well-circumscribed intramuscular tumor, which appears hypointense on T1-weighted and hyperintense on T2-weighted images. The tumor usually appears homogeneous, but heterogeneous presentation due to fibrous septa is reported as well. Concerning the contrast enhancing, three different patterns are described (peripheral enhancement, peripheral and patchy internal enhancement

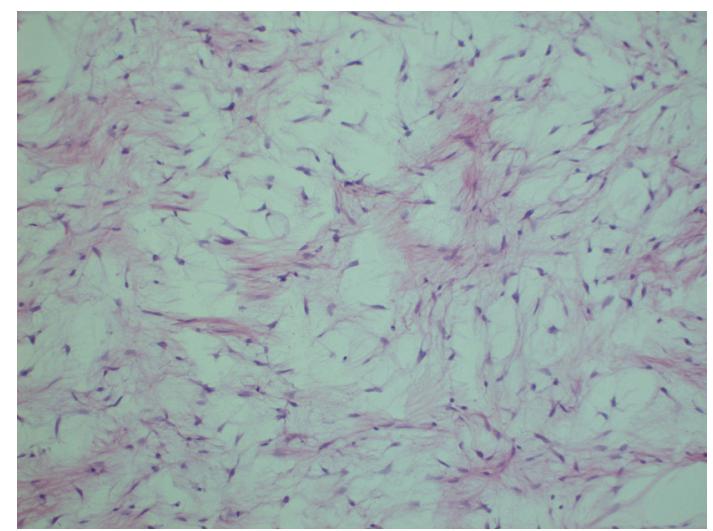

Figure 2. Myxoid tumor with low cell density (HE staining, magnification $\times 10)$. and peripheral and linear internal enhancement) [5].

The issue that makes this case interesting for neurosurgeons is the direct adjacency of the tumor to the sciatic nerve which led to the suspected diagnosis of a PNST with potential malignant behavior due to rapid growth just over few months and influenced the surgical strategy sustainably. Despite the maintenance of recognizable MRI features of intramuscular myxoma [2], the diagnosis was unclear in our case until the end.

\section{References}

1. Allen PW. Myxoma is not a single entity: a review of the concept of myxoma. Ann Diagn Pathol. 2000;4(2):99123.

2. Luna A, Martinez S, Bossen E. Magnetic resonance imaging of intramuscular myxoma with histological comparison and a review of the literature. Skeletal Radiol. 2005;34(1):19-28

3. Nielsen GP, O'Connell JX, Rosenberg AE. Intramuscular myxoma: a clinicopathologic study of 51 cases with emphasis on hypercellular and hypervascular variants. Am J Surg Pathol. 1998;22(10):1222-1227.

4. Silver WP, Harrelson JM, Scully SP. Intramuscular myxoma: a clinicopathologic study of 17 patients. Clin Orthop Relat Res. 2002403):191-197.

5. van Roggen JF, McMenamin ME, Fletcher CD. Cellular myxoma of soft tissue: a clinicopathological study of 38 cases confirming indolent clinical behaviour. Histopathology. 2001;39(3):287-297. 